\title{
Enhancing versus Suppressive Effects of Stress on Immune Function: Implications for Immunoprotection versus Immunopathology
}

\author{
Firdaus S. Dhabhar, PhD
}

It is widely believed that stress suppresses immune function and increases susceptibility to infections and cancer. Paradoxically, stress is also known to exacerbate allergic, autoimmune, and inflammatory diseases. These observations suggest that stress may have bidirectional effects on immune function, being immunosuppressive in some instances and immunoenhancing in others. It has recently been shown that in contrast to chronic stress that suppresses or dysregulates immune function, acute stress can be immunoenhancing. Acute stress enhances dendritic cell, neutrophil, macrophage, and lymphocyte trafficking, maturation, and function and has been shown to augment innate and adaptive immune responses. Acute stress experienced prior to novel antigen exposure enhances innate immunity and memory T-cell formation and results in a significant and long-lasting immunoenhancement. Acute stress experienced during antigen reexposure enhances secondary/adaptive immune responses. Therefore, depending on the conditions of immune activation and the immunizing antigen, acute stress may enhance the acquisition and expression of immunoprotection or immunopathology. In contrast, chronic stress dysregulates innate and adaptive immune responses by changing the type 1-type 2 cytokine balance and suppresses immunity by decreasing leukocyte numbers, trafficking, and function. Chronic stress also increases susceptibility to skin cancer by suppressing type 1 cytokines and protective $T$ cells while increasing suppressor T-cell function. We have suggested that the adaptive purpose of a physiologic stress response may be to promote survival, with stress hormones and neurotransmitters serving as beacons that prepare the immune system for potential challenges (eg, wounding or infection) perceived by the brain (eg, detection of an attacker). However, this system may exacerbate immunopathology if the enhanced immune response is directed against innocuous or self-antigens or dysregulated following prolonged activation, as seen during chronic stress. In view of the ubiquitous nature of stress and its significant effects on immunoprotection and immunopathology, it is important to further elucidate the mechanisms mediating stress-immune interactions and to meaningfully translate findings from bench to bedside.

Key words: allergy, catecholamines, glucocorticoid/cortisol, immune surveillance, proinflammatory/autoimmune, psycho-neuroimmunology, vaccine

P sychological stress is known to suppress immune function and increase susceptibility to infections and cancer. Paradoxically, stress is also known to exacerbate some allergic, autoimmune, and inflammatory diseases, which suggests that stress may enhance immune function under

Firdaus S. Dhabhar: Stanford Center on Stress \& Health and Department of Psychiatry \& Behavioral Sciences, Stanford University, Stanford, CA.

The work described here was supported by grants from the National Institutes of Health (AI48995 and CA107498) and The Dana Foundation.

Correspondence to: Dr. Firdaus S. Dhabhar, Stanford Center on Stress \& Health, Stanford University, 1201 Welch Road, MSLS Building, P114, Stanford, CA 94305-5485; e-mail: dhabhar@rockefeller.edu.

DOI 10.2310/7480.2008.00001 certain conditions. It has recently been appreciated that whereas chronic stress suppresses or dysregulates immune function, acute stress often has immunoenhancing effects. ${ }^{1}$

One of the most underappreciated effects of stress on the immune system is its ability to induce significant changes in leukocyte distribution in the body. ${ }^{2}$ Importantly, these changes have significant effects on immune function in different body compartments that are either enriched or depleted of leukocytes during stress. Moreover, acute stress can affect dendritic cell, neutrophil, macrophage, and lymphocyte trafficking, maturation, or function in ways that can enhance innate and adaptive immunity. ${ }^{3-6}$ Acute stress experienced prior to novel cutaneous antigen exposure increases memory $\mathrm{T}$-cell formation and results in a significant and long-lasting increase in immunity. ${ }^{3,4,6}$ Similarly, acute stress experi- 
enced during antigen reexposure enhances secondary immune responses. ${ }^{7}$ This suggests that depending on the condition under which the immune response is initiated, stress can enhance the acquisition and expression of immunoprotection and immunopathology.

In contrast to acute stress, chronic stress suppresses or dysregulates innate and adaptive immune responses through mechanisms that involve suppression of leukocyte numbers, trafficking, and function or changes in the type 1-type 2 cytokine balance. ${ }^{8,9}$ Chronic stress has recently been shown to increase susceptibility to skin cancer by suppressing type 1 cytokines and protective $\mathrm{T}$ cells while increasing suppressor T-cell function. ${ }^{10}$

We have suggested that the primary biologic purpose of a psychophysiological stress response may be to promote survival, with stress hormones and neurotransmitters serving as beacons that prepare the immune system for potential challenges (eg, wounding or infection) perceived by the brain (eg, detection of an imminent attack). ${ }^{1,2}$ However, this same system may exacerbate immunopathology if the enhanced immune response is directed against innocuous or selfantigens or if the stress response system is overactivated, as seen during chronic stress. In view of the ubiquitous nature of stress and its significant effects on immunoprotection and immunopathology, it is important to further elucidate the mechanisms mediating stress-immune interactions and to translate findings from bench to bedside.

\section{Stress}

Although the word stress generally has negative connotations, stress is a familiar aspect of life, being a stimulant for some but a burden for others. Numerous definitions have been proposed for the word stress. Each definition focuses on aspects of an internal or external challenge, disturbance, or stimulus; on perception of a stimulus by an organism; or on a physiologic response of the organism to the stimulus. ${ }^{11-13}$ Physical stressors have been defined as external challenges to homeostasis and psychological stressors as the "anticipation justified or not, that a challenge to homeostasis looms." ${ }^{14}$ An integrated definition states that stress is a constellation of events, consisting of a stimulus (stressor) that precipitates a reaction in the brain (stress perception) that activates physiologic fight or flight systems in the body (stress response). ${ }^{15}$ The physiologic stress response results in the release of neurotransmitters and hormones that serve as the brain's alarm signals to the body. It is often overlooked that a stress response has salubrious adaptive effects in the short run, ${ }^{1,3}$ although stress can be harmful when it is long-lasting., $8,13,16,17$
An important distinguishing characteristic of stress is its duration and intensity. Thus, acute stress has been defined as stress that lasts for a period of minutes to hours and chronic stress as stress that persists for several hours per day for weeks or months. ${ }^{15}$ The intensity of stress may be gauged by the peak levels of stress hormones, neurotransmitters, and other physiologic changes, such as increases in heart rate and blood pressure, and by the amount of time for which these changes persist during stress and following the cessation of stress.

It is important to bear in mind that significant individual differences exist in the manner and extent to which stress is perceived, processed, and coped with. ${ }^{1}$ These differences become particularly relevant while studying human subjects because stress perception, processing, and coping mechanisms can have significant effects on the kinetics and peak levels of circulating stress hormones and on the duration for which these hormone levels are elevated. The magnitude and duration of catecholamine and glucocorticoid hormone exposure, in turn, can have significant effects on leukocyte distribution and function. ${ }^{18-20}$

\section{Stress-Induced Changes in Immune Cell Distribution}

Effective immunoprotection requires rapid recruitment of leukocytes into sites of surgery, wounding, infection, or vaccination. Immune cells circulate continuously on surveillance pathways that take them from the blood, through various organs, and back into the blood. This circulation is essential for the maintenance of an effective immune defense network. ${ }^{21}$ The numbers and proportions of leukocytes in the blood provide an important representation of the state of distribution of leukocytes in the body and of the state of activation of the immune system. The ability of acute stress to induce changes in leukocyte distribution within different body compartments is perhaps one of the most underappreciated effects of stress and stress hormones on the immune system. ${ }^{2}$

Numerous studies have shown that stress and stress hormones induce significant changes in absolute numbers and relative proportions of leukocytes in the blood. In fact, changes in blood leukocyte numbers were used as a measure of stress before methods were available to directly assay the hormone. ${ }^{22}$ Studies have also shown that glucocorticoid $^{23-25}$ and catecholamine hormones ${ }^{26-31}$ induce rapid and significant changes in leukocyte distribution and that these hormones are the major mediators of the effects of stress. Stress-induced changes in blood 
leukocyte numbers have been reported in fish, ${ }^{32}$ hamsters, ${ }^{33}$ mice, ${ }^{34}$ rats, ${ }^{2,25,35,36}$ rabbits, ${ }^{37}$ horses, ${ }^{38}$ nonhuman primates, ${ }^{39}$ and humans. ${ }^{29,40-43}$ This suggests that the phenomenon of stress-induced leukocyte redistribution has a long evolutionary lineage and that it has important functional significance.

Studies in rodents have shown that stress-induced changes in blood leukocyte numbers are characterized by a significant decrease in the numbers and percentages of lymphocytes and monocytes and by an increase in the numbers and percentages of neutrophils. ${ }^{2,35}$ Flow cytometric analyses revealed that absolute numbers of peripheral blood $\mathrm{T}$ cells, B cells, natural killer (NK) cells, and monocytes all show a rapid and significant decrease (40 to $70 \%$ lower than baseline) during stress. ${ }^{2}$ Moreover, it has been shown that stress-induced changes in leukocyte numbers are rapidly reversed on the cessation of stress. ${ }^{2}$ In apparent contrast to animal studies, human studies have shown that stress can increase rather than decrease blood leukocyte numbers. ${ }^{11,43-46}$ This apparent contradiction may be resolved by taking the following factors into consideration: First, stress-induced increases in blood leukocyte numbers are observed following stress conditions that primarily result in the activation of the sympathetic nervous system. These stressors are often of a short duration (few minutes) or relatively mild (eg, public speaking). ${ }^{1,44-46}$ Second, the increase in total leukocyte numbers may be accounted for by stress- or catecholamine-induced increases in granulocytes and NK cells. ${ }^{8,41,44-46}$ Third, stress- or pharmacologically induced increases in glucocorticoid hormones induce a significant decrease in blood lymphocyte and monocyte numbers. ${ }^{1,25,41,47}$ Thus, stress conditions that result in a significant and sustained activation of the hypothalamic-pituitary-adrenal (HPA) axis result in a decrease in blood leukocyte numbers.

In view of the above discussion, it has been proposed that acute stress induces an initial increase followed by a decrease in blood leukocyte numbers. Stress conditions that result in activation of the sympathetic nervous system, especially conditions that induce high levels of norepinephrine, may induce an increase in circulating leukocyte numbers. These conditions may occur during the beginning of a stress response, very short-duration stress (order of minutes), mild psychological stress, or exercise. In contrast, stress conditions that result in the activation of the HPA axis induce a decrease in circulating leukocyte numbers. These conditions often occur during the later stages of a stress response, long-duration acute stressors (order of hours), or severe psychological, physical, or physiologic stress. An elegant and interesting example in support of this hypothesis comes from Schedlowski and colleagues, who measured changes in blood T-cell and NK cell numbers as well as plasma catecholamine and cortisol levels in parachutists. ${ }^{41}$ Measurements were made 2 hours before, immediately after, and 1 hour after the jump. The results showed a significant increase in T-cell and NK cell numbers immediately (minutes) after the jump that was followed by a significant decrease 1 hour after the jump. An early increase in plasma catecholamines preceded early increases in lymphocyte numbers, whereas the more delayed rise in plasma cortisol preceded the late decrease in lymphocyte numbers. ${ }^{41}$ Importantly, changes in NK cell activity and antibody-dependent cell-mediated cytotoxicity closely paralleled changes in blood NK cell numbers, thus suggesting that changes in leukocyte numbers may be an important mediator of apparent changes in leukocyte "activity." Similarly, Rinner and colleagues showed that a short stressor (1-minute handling) induced an increase in mitogen-induced proliferation of $\mathrm{T}$ and $\mathrm{B}$ cells obtained from peripheral blood, whereas a longer stressor (2-hour immobilization) induced a decrease in the same proliferative responses. ${ }^{48}$ In another example, Manuck and colleagues showed that acute psychological stress induced a significant increase in blood cytotoxic $\mathrm{T}$ lymphocyte numbers only in those subjects who showed heightened catecholamine and cardiovascular reactions to stress. ${ }^{49}$

Thus, an acute stress response may induce biphasic changes in blood leukocyte numbers. Soon after the beginning of stress (order of minutes) or during mild acute stress or exercise, catecholamine hormones and neurotransmitters induce the body's "soldiers" (leukocytes) to exit their "barracks" (spleen, lung, marginated pool, and other organs) and enter the "boulevards" (blood vessels and lymphatics). This results in an increase in blood leukocyte numbers, the effect being most prominent for NK cells and granulocytes. As the stress response continues, activation of the HPA axis results in the release of glucocorticoid hormones, which induce leukocytes to exit the blood and take position at potential "battle stations" (such as the skin, lung, gastrointestinal and urinary-genital tracts, mucosal surfaces, and lymph nodes) in preparation for immune challenges, which may be imposed by the actions of the stressor., ${ }^{2,718}$ Such a redistribution of leukocytes results in a decrease in blood leukocyte numbers. Thus, acute stress may result in a redistribution of leukocytes from the barracks, through the boulevards, and to potential battle stations within the body.

Since the blood is the most accessible and commonly used compartment for human studies, it is important to carefully evaluate how changes in blood immune parameters might reflect in vivo immune function in the 
context of the specific experiments or study at hand. Moreover, since most blood collection procedures involve a certain amount of stress, since all patients or subjects will have experienced acute and chronic stress, and since many studies of psychophysiologic effects on immune function focus on stress, the effects of stress on blood leukocyte distribution become a factor of considerable importance.

Dhabhar and colleagues were the first to propose that stress-induced changes in blood leukocyte distribution may represent an adaptive response. ${ }^{35,50}$ They suggested that acute stress-induced changes in blood leukocyte numbers represent a redistribution of leukocytes from the blood to organs such as the skin, draining sentinel lymph nodes, and other compartments. ${ }^{718}$ They hypothesized that such a leukocyte redistribution may enhance immune function in compartments to which immune cells traffic during stress. In agreement with this hypothesis, it was demonstrated that a stress-induced redistribution of leukocytes from the blood to the skin is accompanied by a significant enhancement of skin immunity. ${ }^{7,50,51}$

\section{Functional Consequences of Stress-Induced Changes in Immune Cell Distribution}

When interpreting data showing stress-induced changes in functional assays such as lymphocyte proliferation or NK activity, it may be important to bear in mind the effects of stress on the leukocyte composition of the compartment in which an immune parameter is being measured. For example, it has been shown that acute stress induces a redistribution of leukocytes from the blood to the skin and that this redistribution is accompanied by a significant enhancement of skin cell-mediated immunity. ${ }^{3,7}$ In what might at first glance appear to be contradicting results, acute stress has been shown to suppress splenic and peripheral blood responses to T-cell mitogens ${ }^{52}$ and splenic immunoglobulin $\mathrm{M}$ ( $\mathrm{IgM})$ production. ${ }^{53}$ However, it is important to note that in contrast to the skin that is enriched in leukocytes during acute stress, peripheral blood and spleen are relatively depleted of leukocytes during acute stress. ${ }^{54}$ This stress-induced decrease in blood and spleen leukocyte numbers may contribute to the acute stress-induced suppression of immune function in these compartments.

Moreover, in contrast to acute stress, chronic stress has been shown to suppress skin cell-mediated immunity, and a chronic stress-induced suppression of blood leukocyte redistribution is thought to be one of the factors mediating the immunosuppressive effect of chronic stress. ${ }^{15}$ Again, in what might appear to be contradicting results, chronic stress has been shown to enhance mitogen-induced proliferation of splenocytes ${ }^{55}$ and splenic IgM production. ${ }^{53}$ However, the spleen is relatively enriched in $\mathrm{T}$ cells during chronic glucocorticoid administration, suggesting that it may also be relatively enriched in $\mathrm{T}$ cells during chronic stress, ${ }^{56}$ and this increase in spleen leukocyte numbers may contribute to the chronic stress-induced enhancement of immune parameters measured in the spleen.

It is also important to bear in mind that the heterogeneity of the stress-induced changes in leukocyte distribution $^{2}$ suggests that using equal numbers of leukocytes in a functional assay may not account for stress-induced changes in relative percentages of different leukocyte subpopulations in the cell suspension being assayed. For example, samples that have been equalized for absolute numbers of total blood leukocytes from control versus stressed animals may still contain different numbers of specific leukocyte subpopulations (eg, T cells, B cells, or NK cells). Such changes in leukocyte composition may contribute to the effects of stress even in functional assays using equalized numbers of leukocytes from different treatment groups. Therefore, stress may affect immune function at a cellular level (eg, phagocytosis, antigen presentation, killing, antibody production) and/or through leukocyte redistribution that could increase or decrease the number of cells with a specific functional capacity in the compartment being studied.

\section{Effects of Acute Stress on Leukocyte Trafficking to a Site of Surgery or Immune Activation}

Viswanathan and colleagues used a clinically relevant subcutaneously implanted surgical sponge model to elucidate the effects of stress on the kinetics, magnitude, subpopulation, and chemoattractant specificity of leukocyte trafficking to a site of immune activation or surgery. ${ }^{5}$ Mice that were acutely stressed before subcutaneous implantation of the surgical sponge showed a two- to threefold higher neutrophil, macrophage, NK cell, and Tcell infiltration than nonstressed animals. Leukocyte infiltration was evident as early as 6 hours and peaked between 24 and 48 hours. Importantly, at 72 hours, sponges from nonstressed and acutely stressed mice had comparable and significantly lower leukocyte numbers, indicating effective resolution of inflammation in both groups. These authors also examined the effects of stress on early (6 hours) leukocyte infiltration in response to a predominantly proinflammatory cytokine, tumour necrosis factor- $\alpha$ (TNF- $\alpha$ ), and lymphocyte-specific chemokine, 
lymphotactin (LTN). Acute stress significantly increased infiltration of macrophages in response to saline, LTN, or TNF- $\alpha$; neutrophils only in response to TNF- $\alpha$; and NK and $\mathrm{T}$ cells only in response to LTN. These results showed that acute stress significantly enhances the kinetics and magnitude of leukocyte infiltration into a site of immune activation or surgery in a subpopulation and chemoattractant-specific manner, with tissue damage or antigenor pathogen-driven chemoattractants synergizing with acute stress to further determine the specific subpopulations that are recruited. ${ }^{5}$ Thus, depending on the primary chemoattractants driving an immune response, acute stress may selectively mobilize specific leukocyte subpopulations into sites of surgery, wounding, or inflammation. Such a stress-induced increase in leukocyte trafficking may be an important mechanism by which acute stressors alter the course of different (innate versus adaptive, early versus late, acute versus chronic) protective or pathologic immune responses.

\section{Acute Stress-Induced Enhancement of Innate/ Primary Immune Responses}

In view of the skin being one of the target organs to which leukocytes traffic during stress, studies were conducted to examine whether skin immunity is enhanced when immune activation/antigen exposure occurs following a stressful experience. Studies showed that acute stress experienced at the time of novel or primary antigen exposure results in a significant enhancement of the ensuing skin immune response. ${ }^{3}$ Compared with controls, mice restrained for 2.5 hours before primary immunization with keyhole limpet hemocyanin (KLH) showed a significantly enhanced immune response when reexposed to KLH 9 months later. This immunoenhancement was mediated by an increase in the numbers of memory and effector helper $\mathrm{T}$ cells in sentinel lymph nodes at the time of primary immunization. Further analyses showed that the early stress-induced increase in T-cell memory may have stimulated the robust increase in infiltrating lymphocyte and macrophage numbers observed months later at a novel site of antigen reexposure. Enhanced leukocyte infiltration was driven by increased levels of the type 1 cytokines interleukin (IL)-2, interferon (IFN)- $\gamma$, and TNF$\alpha$, observed at the site of antigen reexposure in animals that had been stressed at the time of primary immunization. Given the importance of inducing long-lasting increases in immunologic memory during vaccination, it has been suggested that the neuroendocrine stress response is nature's adjuvant, which could be psychologically and/or pharmacologically manipulated to safely increase vaccine efficacy.

In a series of elegant experiments, Saint Mezard and colleagues similarly showed that acute stress experienced at the time of sensitization resulted in a significant increase in the contact hypersensitivity (CHS) response. ${ }^{6}$ These investigators showed that acute stress experienced during sensitization enhanced dendritic cell migration from skin to sentinel lymph nodes and also enhanced priming of lymph node $\mathrm{CD}^{+} \mathrm{T}$ cells. These $\mathrm{CD} 8^{+} \mathrm{T}$ cells responded in greater numbers at the site of antigen reexposure during the recall phase of the CHS response. These studies also suggested that the effects of acute stress in this case were mediated primarily by norepinephrine. ${ }^{6}$

Viswanathan and colleagues further elucidated the molecular and cellular mediators of the immunoenhancing effects of acute stress. ${ }^{57}$ They showed that compared with nonstressed mice, acutely stressed animals showed significantly greater pinna swelling, leukocyte infiltration, and upregulated macrophage chemoattractant protein 1 (MCP-1), macrophage inflammatory protein $3 \alpha$ (MIP$3 \alpha)$, IL- $1 \alpha$, IL- $1 \beta$, IL- 6 , TNF- $\alpha$, and IFN- $\gamma$ gene expression at the site of primary antigen exposure. Stressed animals also showed enhanced maturation and trafficking of dendritic cells from skin to lymph nodes, higher numbers of activated macrophages in skin and lymph nodes, increased T-cell activation in lymph nodes, and enhanced recruitment of surveillance $\mathrm{T}$ cells to skin. These findings showed that important interactive components of innate (dendritic cells and macrophages) and adaptive (surveillance $\mathrm{T}$ cells) immunity are mediators of the stressinduced enhancement of a primary immune response. Such immunoenhancement during primary immunization may induce a long-term increase in immunologic memory, resulting in subsequent augmentation of the immune response during secondary antigen exposure.

In addition to elucidating mechanisms that could be targeted to reduce stress-induced exacerbation of allergic, autoimmune, and proinflammatory reactions, the abovementioned studies provide further support for the idea that a psychophysiologic stress response is nature's fundamental survival mechanism that could be therapeutically harnessed to augment immune function during vaccination, wound healing, or infection.

\section{Acute Stress-Induced Enhancement of Adaptive/ Secondary Immune Responses}

Studies have shown that in addition to enhancing primary cutaneous immune responses, acute stress experienced at 
the time of antigen reexposure can also enhance secondary or recall responses in skin. ${ }^{7}$ Compared with nonstressed controls, mice that were acutely stressed at the time of antigen reexposure showed a significantly larger number of infiltrating leukocytes at the site of the immune reaction. These results demonstrated that a relatively mild behavioural manipulation can enhance an important class of immune responses that mediate harmful (allergic dermatitis) and beneficial (resistance to certain viruses, bacteria, and tumours) aspects of immune function.

Blecha and colleagues reported a similar stressinduced enhancement of CHS reactions in mice, ${ }^{58}$ and Flint and colleagues showed that acute stress enhanced CHS responses in both male and female mice and that immunoenhancement was partially dependent on glucocorticoid hormones. ${ }^{59}$ Stress-induced enhancement of the elicitation phase of skin cell-mediated immunity has also been reported in hamsters. ${ }^{33}$ Taken together, studies show that acute stress can significantly enhance the immunization/sensitization/induction, as well as the reexposure/elicitation/recall phases of skin cell-mediated immunity.

\section{Hormone and Cytokine Mediators of Stress-Induced Enhancement of Immune Function}

Although much work remains to be done to identify molecular, cellular, and physiologic mechanisms mediating the adjuvant-like, immunoenhancing effects of acute stress, several studies have begun to identify endocrine and immune mediators of these effects. Studies have shown that corticosterone and epinephrine are important mediators of an acute stress-induced immunoenhancement. ${ }^{50}$ Adrenalectomy, which eliminates the glucocorticoid and epinephrine stress response, eliminated the stress-induced enhancement of skin delayed-type hypersensitivity (DTH). Low-dose corticosterone or epinephrine administration significantly enhanced skin DTH. ${ }^{50}$ In contrast, high-dose corticosterone, chronic corticosterone, or low-dose dexamethasone administration significantly suppressed skin DTH. These results suggested a novel role for adrenal stress hormones as endogenous immunoenhancing agents. They also showed that stress hormones released during a circumscribed or acute stress response may help prepare the immune system for potential challenges (eg, wounding or infection), for which stress perception by the brain may serve as an early warning signal. Studies by Flint and colleagues have also suggested that corticosterone is a mediator of the stress-induced enhancement of skin CHS, ${ }^{59}$ whereas Saint-Mezard and colleagues have sug- gested that the adjuvant-like effects of stress on dendritic cell and $\mathrm{CD} 8^{+} \mathrm{T}$-cell migration and function are mediated by norepinephrine. ${ }^{6}$

Studies have also examined the immunologic mediators of an acute stress-induced enhancement of skin immunity. Since IFN- $\gamma$ is a critical cytokine mediator of cell-mediated immunity and delayed as well as contact hypersensitivity, studies were conducted to examine its role as a local mediator of the stress-induced enhancement of skin DTH. ${ }^{51}$ The effect of acute stress on skin DTH was examined in wild-type and IFN- $\gamma$ receptor gene knockout mice (IFN- $\gamma \mathrm{R}^{-/-}$) that had been sensitized with 2,4-dinitro-1-fluorobenzene (DNFB). Acutely stressed wild-type mice showed a significantly larger DTH response than nonstressed mice. In contrast, IFN- $\gamma \mathrm{R}^{-1-}$ mice failed to show a stress-induced enhancement of skin DTH. Immunoneutralization of IFN- $\gamma$ in wild-type mice significantly reduced the stress-induced enhancement of skin DTH. In addition, an inflammatory response to direct IFN- $\gamma$ administration was significantly enhanced by acute stress. These results showed that IFN- $\gamma$ is an important local mediator of a stress-induced enhancement of skin DTH. ${ }^{51}$ In addition to IFN- $\gamma$, TNF- $\alpha$ MCP-1, MIP- $3 \alpha$, IL-1, and IL-6 have also been associated with a stress-induced enhancement of the immunization/ sensitization phase of skin cell-mediated immunity. ${ }^{3,57}$ It is clear that further investigation is necessary to identify the most important molecular, cellular, and physiologic mediators of a stress-induced enhancement of skin immunity.

\section{Stress-Induced Suppression of Immune Function}

In contrast to acute stressors, chronic stress has been shown to suppress or dysregulate immune function. ${ }^{8,60-66}$ Dhabhar and colleagues conducted studies designed to examine the effects of increasing the intensity and duration of acute stress, as well as the transition from acute to chronic stress on skin immune function. ${ }^{15}$ These studies showed that acute stress administered for 2 hours prior to antigenic challenge significantly enhanced skin cellmediated immunity. ${ }^{15}$ Increasing the duration of stress from 2 to 5 hours produced the same magnitude of immunoenhancement. Interestingly, increasing the intensity of acute stress produced a significantly larger enhancement of the DTH response, which was accompanied by increasing magnitudes of leukocyte redeployment. In contrast, these studies found suppression of the skin immune response when chronic stress exposure was begun 3 weeks before sensitization and either discontinued 
on sensitization, or continued an additional week until challenge, or extended for 1 week after challenge. ${ }^{15}$ Interestingly, acute stress-induced redistribution of peripheral blood lymphocytes was attenuated with increasing duration of stressor exposure and correlated with attenuated glucocorticoid responsivity. These results suggested that stress-induced alterations in lymphocyte redeployment may play an important role in mediating the bidirectional effects of stress on cutaneous cell-mediated immunity. ${ }^{5}$ An association between chronic stress and reduced skin cell-mediated immunity has also been reported in human subjects. ${ }^{67}$

Given the importance of cutaneous cell-mediated immunity in elimination of immunoresponsive tumours such as squamous cell carcinoma SCC, ${ }^{68,69}$ Saul and colleagues examined the effects of chronic stress on susceptibility to ultraviolet radiation (UV)-induced SCC. ${ }^{10}$ Mice were exposed to a minimal erythemal dose of UVB three times a week for 10 weeks. Half of the UVBexposed mice were left nonstressed (ie, they remained in their home cages), and the other half were chronically stressed (ie, restrained during weeks 4-6). UV-induced tumours were measured weekly from week 11 through week 34 , blood was collected at week 34 , and tissues were collected at week 35 . Messenger ribonucleic acid expression of IL-12p40, IFN- $\gamma$, IL-4, IL-10, CD3 $\varepsilon$, and CCL27/ CTACK, the skin T cell-homing chemokine, in dorsal skin was quantified using real-time polymerase chain reaction. $\mathrm{CD}^{+}, \mathrm{CD}^{+}$, and $\mathrm{CD} 25^{+}$leukocytes were counted using immunohistochemistry and flow cytometry. Stressed mice had a shorter median time to first tumour ( 15 vs 16.5 weeks) and reached 50\% incidence earlier than controls ( 15 weeks vs 21 weeks). Stressed mice also had lower IFN$\gamma$, CCL27/CTACK, and CD3 $\varepsilon$ gene expression and lower $\mathrm{CD}^{+}$and $\mathrm{CD}^{+}{ }^{+} \mathrm{T}$ cells infiltrating within and around tumours than nonstressed mice. In addition, stressed mice had higher numbers of tumour infiltrating and circulating $\mathrm{CD} 4^{+} \mathrm{CD} 25^{+}$suppressor cells than nonstressed mice. These studies showed that chronic stress increased susceptibility to UV-induced SCC by suppressing skin immunity, type 1 cytokines, and protective $\mathrm{T}$ cells and increasing active immunosuppression through regulatory/suppressor $\mathrm{T}$ cells. $^{10}$

Similarly, in human and animal studies, chronic stress has also been shown to suppress different immune parameters, examples of which include $\mathrm{CMI},{ }^{70,71}$ antibody production, ${ }^{72} \mathrm{NK}$ activity, ${ }^{16,74-76}$ leukocyte proliferation, ${ }^{74,75,77}$ skin homograft rejection, ${ }^{78}$ virus-specific Tcell and NK cell activity, ${ }^{79}$ and antimycobacterial activity of macrophages from susceptible mouse strains. ${ }^{80}$

\section{Stress-Immune Spectrum}

Dhabhar and McEwen have proposed that a stress response and its effects on immune function be viewed in the context of a stress-immune spectrum. ${ }^{1,15,18}$ One region of this spectrum is characterized by acute stress or eustress, that is, conditions of short-duration stress that may result in immunopreparatory or immunoenhancing physiologic conditions. An important characteristic of acute stress is a rapid physiologic stress response mounted in the presence of the stressor, followed by a rapid shutdown of the response upon cessation of stress. The other end of the stress spectrum is characterized by chronic stress or distress, that is, repeated or prolonged stress that may result in dysregulation or suppression of immune function. An important characteristic of distress is that the physiologic response either persists long after the stressor has ceased or is activated repeatedly to result in an overall integrated increase in exposure of the organism to stress hormones. The concept of "allostatic load" has been proposed to define the "psychophysiological wear and tear" that takes place while different biologic systems work to stay within a range of equilibrium (allostasis) in response to demands placed by internal or external chronic stressors. ${ }^{12,17}$ We suggest that conditions of high allostatic load would result in dysregulation or suppression of immune function. Importantly, a disruption of the circadian corticosterone/cortisol rhythm may be an indicator and/or mediator of distress or high allostatic load. ${ }^{15,81}$ The stress-immune spectrum also proposes that between eustress and distress is a region that represents resilience, which we define as the ability of physiologic systems to enable survival under increasingly challenging conditions. The psychophysiologic mechanisms of resilience ${ }^{82}$ provide an attractive area for future research.

The stressor itself can be acute or chronic. Importantly, cognitive mechanisms mediating stress perception, coping, and sense of control and psychosocial factors such as social support are critical determinants of the duration and magnitude of a physiologic stress response that is driven by the brain for any given stressor. Cognitive perception and coping mechanisms are especially important in humans because they can psychologically "convert" acute stressors into chronic physiologic stress responses. Psychogenic stress is also a very important phenomenon in human subjects because it can generate physiologic stress responses long after stressor exposure (eg, lingering anger/mood disturbance following a social altercation) or even in the absence of a physical stressor or salient threat 
Table 1. Factors that Determine whether Psychological Stress May Enhance or Suppress Immune Function

\begin{tabular}{lll}
\hline Factor & \multicolumn{1}{c}{$\begin{array}{c}\text { Stress-Induced } \\
\text { Immunoenhancement }\end{array}$} & $\begin{array}{c}\text { Stress-Induced } \\
\text { Immunosuppression }\end{array}$ \\
\hline Immune cell redistribution & $\begin{array}{c}\text { In leukocyte-enriched compartments } \\
\text { (eg, skin, sentinel lymphnodes) } \\
\text { Acute (minutes to hours) }\end{array}$ & $\begin{array}{c}\text { In leukocyte-depleted compartments (eg, blood, } \\
\text { spleen) } \\
\text { Duration of stress or hormone } \\
\text { exposure }\end{array}$ \\
$\begin{array}{l}\text { Concentration of hormone (weeks/months/years) } \\
\text { Source of glucocorticoid }\end{array}$ & $\begin{array}{l}\text { Physiologic stress levels } \\
\text { Endogenous }\end{array}$ & $\begin{array}{l}\text { High pharmacologic levels } \\
\text { Synthetic analogues }\end{array}$ \\
\hline
\end{tabular}

(eg, worrying about whether one's romantic feelings will be reciprocated). Therefore, following stressor exposure and its processing by the brain, a physiologic stress response ensues. This response may consist of acute or chronic physiologic activation (neurotransmitters, hormones, and their molecular, cellular, organ-level, and systemic effects) that has differential effects on immune function.

The duration, magnitude, and timing of exposure to stress or stress hormones and the source (endogenous versus synthetic) of hormone are critical for determining whether, in a given situation, stress will enhance or suppress/dysregulate immune function (Table 1).

\section{Conclusion}

An important function of physiologic mediators released under conditions of acute psychological stress may be to ensure that appropriate leukocytes are present in the right place and at the right time to respond to an immune challenge that might be initiated by the stress-inducing agent (eg, attack by a predator, invasion by a pathogen, etc.). The modulation of immune cell distribution by acute stress may be an adaptive response designed to enhance surveillance and increase the capacity of the immune system to respond to challenge in compartments (such as the skin, lung, gastrointestinal and urinary-genital tracts, mucosal surfaces, and lymph nodes), which serve as defense barriers for the body. Thus, neurotransmitters and hormones released during stress may increase immunosurveillance and help enhance immune preparedness for potential (or ongoing) immune challenge. Stress-induced immunoenhancement may increase immunoprotection during surgery, vaccination, or infection but may also exacerbate immunopathology during inflammatory (asthma, allergy, dermatitis, cardiovascular disease, gingivitis) or autoimmune (psoriasis, arthritis, multiple sclerosis) diseases that are known to be exacerbated by stress. ${ }^{83-88}$
The relationships between immune function and the physiologic manifestations of stress are complex. The studies described here shed light on potential mechanisms that may mediate the bidirectional effects of stress on immune function and provide substrates for interventions that may be designed to dampen or eliminate stress-induced exacerbation of allergic, inflammatory, or autoimmune diseases. Although decades of research have examined the pathologic effects of stress on immune function and on health, the study of salubrious or health-promoting effects of stress is relatively new. ${ }^{1,3}$ Therefore, the studies presented here also provide a framework for developing therapeutic interventions that harness the mind and body's endogenous health-promoting mechanisms to enhance protective immunity during vaccination, infection, or wound healing. Much work remains to be done to elucidate the mechanisms mediating the salubrious versus health-aversive effects of stress and to translate basic findings in the field from bench to bedside. Moreover, this work is extremely important because stress is a ubiquitous aspect of life and is thought to play a role in the etiology of numerous diseases.

\section{Acknowledgements}

I wish to thank current and previous members of my laboratory, particularly Dr. Kavitha Viswanathan, Dr. Alison Saul, Kanika Ghai, Christine Daugherty, and Jean Tillie, whose work and publications are among those that are discussed in this chapter.

\section{References}

1. Dhabhar FS, McEwen BS. Bidirectional effects of stress on immune function: possible explanations for salubrious as well as harmful effects. In: Ader R, editor. Psychoneuroimmunology. 4th ed. San Diego (CA): Elsevier; 2006.

2. Dhabhar FS, Miller AH, McEwen BS, Spencer RL. Effects of stress on immune cell distribution-dynamics and hormonal mechanisms. J Immunol 1995;154:5511-27. 
3. Dhabhar FS, Viswanathan K. Short-term stress experienced at the time of immunization induces a long-lasting increase in immunological memory. Am J Physiol Regul Integr Comp Physiol 2005; 289:R738-744.

4. Viswanathan K, Daugherty C, Dhabhar FS. Stress as an endogenous adjuvant: augmentation of the immunization phase of cell-mediated immunity. Int Immunol 2005;17:1059-69.

5. Viswanathan K, Dhabhar FS. Stress-induced enhancement of leukocyte trafficking into sites of surgery or immune activation. PNAS 2005;102:5808-13.

6. Saint-Mezard P, Chavagnac C, Bosset S, et al. Psychological stress exerts an adjuvant effect on skin dendritic cell functions in vivo. J Immunol 2003;171:4073-80.

7. Dhabhar FS, McEwen BS. Stress-induced enhancement of antigenspecific cell-mediated immunity. J Immunol 1996;156:2608-15.

8. Glaser R, Kiecolt-Glaser JK. Stress-induced immune dysfunction: implications for health. Nat Rev Immunol 2005;5:243-51.

9. Ader R. Psychoneuroimmunology. 4th ed. San Diego (CA): Academic Press; 2006.

10. Saul AN, Oberyszyn TM, Daugherty C, et al. Chronic stress and susceptibility to skin cancer. J Natl Cancer Inst 2005;97:1760-7.

11. Goldstein DS, McEwen BS. Allostasis, homeostats, and the nature of stress. Stress 2002;5:55-8.

12. McEwen BS. The end of stress as we know it. Washington (DC): Dana Press; 2002.

13. Sapolsky RM. Why zebras don't get ulcers. 3rd ed. W.H. Freeman and Company; 2004.

14. Sapolsky RM. The influence of social hierarchy on primate health. Science 2005;308:648-52.

15. Dhabhar FS, McEwen BS. Acute stress enhances while chronic stress suppresses immune function in vivo: A potential role for leukocyte trafficking. Brain Behav Immun 1997;11:286-306.

16. Irwin $\mathrm{M}$, et al. Reduction of immune function in life stress and depression. Biol Psychiatry 1990;27:22-30.

17. McEwen BS. Protective and damaging effects of stress mediators: allostasis and allostatic load. N Engl J Med 1998;338:171-9.

18. Dhabhar FS, McEwen BS. Bidirectional effects of stress and glucocorticoid hormones on immune function: possible explanations for paradoxical observations. In: Ader R, Felten DL, Cohen N, editors. Psychoneuroimmunology. 3rd ed. San Diego (CA): Academic Press; 2001. p. 301-38.

19. Pruett SB. Quantitative aspects of stress-induced immunomodulation. Int Immunopharmacol 2001;1:507-20.

20. Schwab CL, et al. Modeling and predicting stress-induced immunosuppression in mice using blood parameters. Toxicol Sci 2005;83:101-13.

21. Sprent J, Tough DF. Lymphocyte life-span and memory. Science 1994;265:1395-400.

22. Hoagland H, Elmadjian F, Pincus G. Stressful psychomotor performance and adrenal cortical function as indicated by the lymphocyte reponse. J Clin Endocrinol 1946;6:301-11.

23. Fauci AS, Dale DC. The effect of in vivo hydrocortisone on subpopulations of human lymphocytes. J Clin Invest 1974;53:240-6.

24. Fauci AS, Dale DC. The effect of hydrocortisone on the kinetics of normal human lymphocytes. Blood 1975;46:235-43.

25. Dhabhar FS, Miller AH, McEwen BS, Spencer RL. Stress-induced changes in blood leukocyte distribution-role of adrenal steroid hormones. J Immunol 1996;157:1638-44.
26. Carlson SL, Fox S, Abell KM. Catecholamine modulation of lymphocyte homing to lymphoid tissues. Brain Behav Immun 1997;11:307-20.

27. Benschop RJ, et al. Beta 2-adrenergic stimulation causes detachment of natural killer cells from cultured endothelium. Eur J Immunol 1993;23:3242-7.

28. Benschop RJ, Rodriguez-Feuerhahn $\mathrm{M}$, Schedlowski $\mathrm{M}$. Catecholamine-induced leukocytosis: early observations, current research, and future directions. Brain Behav Immun 1996;10:77-91.

29. Mills PJ, et al. Catecholamines, catecholamine receptors, cell adhesion molecules, and acute stressor-related changes in cellular immunity. Adv Pharmacol 1998;42:587-90.

30. Redwine L, et al. Acute psychological stress: effects on chemotaxis and cellular adhesion molecule expression. Psychosom Med 2003; 65:598-603.

31. Mills PJ, et al. Peripheral leukocyte subpopulations and catecholamine levels in astronauts as a function of mission duration. Psychosom Med 2001;63:886-90.

32. Pickford GE, Srivastava AK, Slicher AM, Pang PKT. The stress response in the abundance of circulating leukocytes in the killifish, Fundulus heteroclitus. I The cold-shock sequence and the effects of hypophysectomy. J Exp Zool 1971;177:89-96.

33. Bilbo SD, Dhabhar FS, Viswanathan K, et al. Short day lengths augment stress-induced leukocyte trafficking and stress-induced enhancement of skin immune function. Proc Natl Acad Sci U S A 2002;99:4067-72.

34. Jensen MM. Changes in leukocyte counts associated with various stressors. J Reticuloendothelial Soc 1969;8:457-65.

35. Dhabhar FS, Miller AH, Stein M, et al. Diurnal and stress-induced changes in distribution of peripheral blood leukocyte subpopulations. Brain Behav Immun 1994;8:66-79.

36. Rinder CS, et al. Lymphocyte and monocyte subset changes during cardiopulmonary bypass: effects of aging and gender. J Lab Clin Med 1997;129:592-602.

37. Toft P, Svendsen P, Tonnesen E, et al. Redistribution of lymphocytes after major surgical stress. Acta Anesthesiol Scand 1993;37:245-9.

38. Snow DH, Ricketts SW, Mason DK. Hematological responses to racing and training exercise in thoroughbred horses, with particular reference to the leukocyte response. Equine Vet J 1983; 15:149-54.

39. Morrow-Tesch JL, McGlone JJ, Norman RL. Consequences of restraint stress on natural killer cell activity, behavior, and hormone levels in rhesus macaques (Macaca mulatta). Psychoneuroendocrinology 1993;18:383-95.

40. Herbert TB, Cohen S. Stress and immunity in humans: a metaanalytic review. Psychosom Med 1993;55:364-79.

41. Schedlowski M, Jacobs R, Stratman G, et al. Changes of natural killer cells during acute psychological stress. J Clin Immunol 1993; 13:119-26.

42. Redwine L, et al. Differential immune cell chemotaxis responses to acute psychological stress in Alzheimer caregivers compared to non-caregiver controls. Psychosom Med 2004;66:770-5.

43. Bosch JA, et al. Acute stress evokes selective mobilization of $\mathrm{T}$ cells that differ in chemokine receptor expression: a potential pathway linking immunologic reactivity to cardiovascular disease. Brain Behav Immun 2003;17:251-9.

44. Naliboff BD, et al. Immunological changes in young and old adults during brief laboratory stress. Psychosom Med 1991;53:121-32.

45. Brosschot JF, et al. Influence of life stress on immunological reactivity to mild psychological stress. Psychosom Med 1994;56:216-24. 
46. Mills PJ, et al. Lymphocyte subset redistribution in response to acute experimental stress: effects of gender, ethnicity, hypertension, and the sympathetic nervous system. Brain Behav Immun 1995;9:61-9.

47. Stein M, Ronzoni E, Gildea EF. Physiological responses to heat stress and ACTH of normal and schizophrenic subjects. Am J Psychiatry 1951;6:450-5.

48. Rinner I, Schauenstein K, Mangge H, et al. Opposite effects of mild and severe stress on in vitro activation of rat peripheral blood lymphocytes. Brain Behav Immun 1992;6:130-40.

49. Manuck SB, Cohen S, Rabin BS, et al. Individual differences in cellular immune response to stress. Psychol Sci 1991;2:111-5.

50. Dhabhar FS, McEwen BS. Enhancing versus suppressive effects of stress hormones on skin immune function. PNAS 1999;96:1059-64.

51. Dhabhar FS, Satoskar AR, Bluethmann H, et al. Stress-induced enhancement of skin immune function: a role for IFN $\gamma$. PNAS 2000;97:2846-51.

52. Cunnick JE, Lysle DT, Kucinski BJ, Rabin BS. Evidence that shockinduced immune suppression is mediated by adrenal hormones and peripheral beta-adrenergic receptors. Pharmacol Biochem Behav 1990;36:645-51.

53. Zalcman S, Anisman H. Acute and chronic stressor effects on the antibody response to sheep red blood cells. Pharmacol Biochem Behav 1993;46:445-52.

54. Dhabhar FS. Stress-induced enhancement of cell-mediated immunity. Ann N Y Acad Sci 1998;840:359-72.

55. Monjan AA, Collector MI. Stress-induced modulation of the immune response. Science 1977;196:307-8.

56. Miller AH, Spencer RL, Hasset J, et al. Effects of selective type I and type II adrenal steroid receptor agonists on immune cell distribution. Endocrinology 1994;135:1934-44.

57. Viswanathan K, Daugherty C, Dhabhar FS. Stress as an endogenous adjuvant: augmentation of the immunization phase of cell-mediated immunity. Int Immunol 2005;17:1059-69.

58. Blecha F, Barry RA, Kelley KW. Stress-induced alterations in delayed-type hypersensitivity to SRBC and contact sensitivity to DNFB in mice. Proc Soc Exp Biol Med 1982;169:239-46.

59. Flint MS, Miller DB, Tinkle SS. Restraint-induced modulation of allergic and irritant contact dermatitis in male and female B6.129 mice. Brain Behav Immun 2000;14:256-69.

60. Sklar LS, Anisman H. Stress and cancer. Psychol Bull 1981;89:369-406.

61. Borysenko M, Borysenko J. Stress, behavior, and immunity: animal models and mediating mechanisms. Gen Hosp Psychiatry 1982;4:59-67.

62. Khansari DN, Murgo AJ, Faith RE. Effects of stress on the immune system. Immunol Today 1990;11:170-5.

63. Zwilling B. Stress affects disease outcomes. Confronted with infectious disease agents, the nervous and immune systems interact in complex ways. ASM News 1992;58:23-5.

64. Kort WJ. The effect of chronic stress on the immune system. Adv Neuroimmunol 1994;4:1-11.

65. Irwin M. Stress-induced immune suppression: role of brain corticotropin releasing hormone and autonomic nervous system mechanisms. Adv Neuroimmunol 1994;4:29-47.

66. Black PH. Immune system-central nervous system interactions: effect and immunomodulatory consequences of immune system mediators on the brain. Antimicrob Agents Chemother 1994;38:7-12.

67. Smith A, et al. The relationship between distress and the development of a primary immune response to a novel antigen. Brain Behav Immun 2004;18:65-75.
68. Kripke ML. Ultraviolet radiation and immunology: something new under the sun - presidential address. Cancer Res 1994;54:6102-5.

69. Granstein RD, Matsui MS. UV radiation-induced immunosuppression and skin cancer. Cutis 2004;74(5 Suppl):4-9.

70. Kelley KW, Greenfield RE, Evermann JF, et al. Delayed-type hypersensitivity, contact sensitivity, and PHA skin-test responses of heat- and cold-stressed calves. Am J Vet Res 1982;43:775-9.

71. Basso AM, Depiante-Depaoli M, Cancela L, Molina V. Seven-day variable-stress regime alters cortical $\beta$-adrenoreceptor binding and immunologic responses: reversal by imipramine. Pharmacol Biochem Behav 1993;45:665-72.

72. Edwards EA, Dean LM. Effects of crowding of mice on humoral antibody formation and protection to lethal antigenic challenge. Psychosom Med 1977;39:19-24.

73. Fleshner M, Laudenslager ML, Simons L, Maier SF. Reduced serum antibodies associated with social defeat in rats. Physiol Behav 1989; 45:1183-7.

74. Bartrop R, Lazarus L, Luckhurst E, et al. Depressed lymphocyte function after bereavement. Lancet 1977;8016:834-6.

75. Cheng GJ, Morrow-Tesch JL, Beller DI, et al. Immunosuppression in mice induced by cold water stress. Brain Behav Immunity 1990; 4:278-91.

76. Kiecolt-Glaser JK, et al. Psychosocial modifiers of immunocompetence in medical students. Psychosom Med 1984;46:7-14.

77. Regnier JA, Kelley KW. Heat- and cold-stress suppresses in vivo and in vitro cellular immune response of chickens. Am J Vet Res 1981;42:294-9.

78. Wistar R, Hildemann WH. Effect of stress on skin transplantation immunity in mice. Science 1960;131:159-60.

79. Bonneau RH, Sheridan JF, Feng N, Glaser R. Stress-induced effects on cell-mediated innate and adaptive memory components of the murine immune response to herpes simplex virus infection. Brain Behav Immunity 1991;5:274-95.

80. Brown DH, Zwilling BS. Activation of the hypothalamic-pituitaryadrenal axis differentially affects the anti-mycobacterial activity of macrophages from BCG-resistant and susceptible mice. J Neuroimmunol 1994;53:181-7.

81. Sephton SE, Sapolsky RM, Kraemer HC, Spiegel D. Diurnal cortisol rhythm as a predictor of breast cancer survival. J Natl Cancer Inst 2000;92:994-1000.

82. Charney DS. Psychobiological mechanisms of resilience and vulnerability: implications for successful adaptation to extreme stress. Am J Psychiatry 2004;161:195-216.

83. Amkraut AA, Solomon CF, Kraemer HC. Stress, early experience and adjuvant-induced arthritis in the rat. Psychosom Med 1971;33: 203-14.

84. Ackerman KD, et al. Stressful life events precede exacerbations of multiple sclerosis. Psychosom Med 2002;64:916-20.

85. Al'Abadie MS, Kent GG, Gawkrodger DJ. The relationship between stress and the onset and exacerbation of psoriasis and other skin conditions. Br J Dermatol 1994;130:199-203.

86. Garg A, et al. Psychological stress perturbs epidermal permeability barrier homeostasis: implications for the pathogenesis of stressassociated skin disorders. Arch Dermatol 2001;137:53-9.

87. Wright RJ, Rodriguez M, Cohen S. Review of psychosocial stress and asthma: an integrated biopsychosocial approach. Thorax 1998; 53:1066-74.

88. Wright RJ. Stress and atopic disorders. J Allergy Clin Immunol 2005;116:1301-6. 